\title{
Asymptotic Time-Behaviour of Solutions to Scalar Conservation Law with a Convex Flux Function
}

\author{
Natalia Petrosyan ${ }^{1, *}$ \\ ${ }^{1}$ Moscow State Technological University "STANKIN", RU-127055, Moscow, Russia
}

\begin{abstract}
We consider the long-time behaviour of solutions of the Cauchy problem for a quasilinear equation $u_{t}+f(u)_{x}=0$ with a strictly convex flux function $f(u)$ and initial function $u_{0}(x)$ having the one-sided limiting mean values $u^{ \pm}$that are uniform with respect to translations. The estimates of the rates of convergence to solutions of the Riemann problem depending on the behaviour of the integrals $\int_{a}^{a+y}\left(u_{0}(x)-u^{ \pm}\right) d x$ as $y \rightarrow \pm \infty$ are established. The similar results are obtained for solutions of the mixed problem in the domain $x>0, t>0$ with a constant boundary data $u^{-}$and initial data having limiting mean value $u^{+}$.
\end{abstract}

\section{Introduction}

In the half-plane $\Pi^{+}=(0, \infty) \times R^{1}$ the Cauchy problem is considered for the quasilinear equation

$$
u_{t}+(f(u))_{x}=0, x \in R^{1}, t>0
$$

with the initial condition

$$
\left.u\right|_{t=0}=u_{0}(x), x \in R^{1} .
$$

The articles concerning the asymptotic properties of the generalized entropy solutions of the problem (1), (2) for large values of time are reviewed in [1]. New interesting results are obtained in the last years for the case of non-convex flux function $f(u)$ (see [2], [3], [4] for example).

It is assumed throughout this paper that the function $f(u)$, defined for all values of $u \in R^{1}$, is twice continuously differentiable and convex downward

$$
f(u) \in C^{2}, f^{\prime \prime}(u)>0,
$$

the function $u_{0}(x)$ is bounded and measurable .

Definition ([5]). A bounded measurable function $u(t, x)$ in $\Pi^{+}$is called a generalized solution of problem (1), (2) if the following conditions are satisfied:

$$
\begin{aligned}
& \forall g(t, x) \in C^{\infty}\left(\Pi^{+}\right), g(t, x) \equiv 0 \text { for } t \geq \text { const and } \\
& |x| \geq \text { const }, \\
& \int_{\Pi^{+}}\left\{u \cdot g_{t}+f(u) g_{x}\right\} d x d t+\int_{-\infty}^{+\infty} u_{0}(x) g(0, x) d x=0 ;
\end{aligned}
$$

there exists a constant $C>0$ such that for a.e. $x_{1} \in \mathrm{R}^{1}, x_{2} \in \mathrm{R}^{1}, x_{1} \neq x_{2}$ and $t>0$

$$
\frac{u\left(t, x_{1}\right)-u\left(t, x_{2}\right)}{x_{1}-x_{2}} \leq \frac{C}{t} .
$$

A generalized solution of problem (1), (2) in the sense of this definition is unique and exists for any bounded measurable initial function $u_{0}(x)$ (see [4]).

\section{Cauchy problem}

Assume that the initial function $u_{0}(x)$ has the one-sided limiting mean values that are uniform with respect to translations: $\forall a_{0} \in R^{1}$

$$
\begin{aligned}
& \frac{1}{y} \int_{a}^{a+y} u_{0}(x) d x \rightarrow u^{-} \text {as } y \rightarrow-\infty \text { uniformly in } a \leq a_{0} \text {, } \\
& \frac{1}{y} \int_{a}^{a+y} u_{0}(x) d x \rightarrow u^{+} \text {as } y \rightarrow+\infty \text { uniformly in } a \geq a_{0} \text {. }
\end{aligned}
$$

The following theorems 1 and 2 are proved in [8]. 
Theorem 1. Let (3), (4) holds and $u^{-}>u^{+}$. Then there exists a number $T_{0}>0$ and a function $a(t) \in C\left(\left[T_{0},+\infty\right)\right), a(t) / t \rightarrow 0 \quad$ as $\quad t \rightarrow+\infty$ such that $u(t, x)-S(t, x) \rightarrow 0$ as $t \rightarrow+\infty$ uniformly in $x \in R^{1}$, where

$$
S(t, x)=\left\{\begin{array}{l}
u^{-}, x<k t+a(t), \\
u^{+}, x>k t+a(t),
\end{array} \quad k=\frac{f\left(u^{+}\right)-f\left(u^{-}\right)}{u^{+}-u^{-}} .\right.
$$

Theorem 2. Let (3), (4) holds and $u^{-}<u^{+}$. Then $u(t, x)-H(x / t) \rightarrow 0$ as $t \rightarrow+\infty$ uniformly in $x \in R^{1}$, where

$$
H(x / t)=\left\{\begin{array}{c}
u^{-}, x<f^{\prime}\left(u^{-}\right) t, \\
f^{\prime-1}(x / t), f^{\prime}\left(u^{-}\right) \leq x / t<f^{\prime}\left(u^{+}\right), \\
u^{+}, x \geq f^{\prime}\left(u^{+}\right) t .
\end{array}\right.
$$

In this paper the rates of convergence of the solution $u(t, x)$ to the limiting functions $S(t, x)$ and $H(x / t)$ are established.

Theorem 3. Assume that $\forall a_{0} \in R^{1}$

$$
\int_{a}^{a+y}\left(u_{0}(x)-u^{-}\right) d x=O\left(|y|^{\alpha^{-}}\right) \text {as } y \rightarrow-\infty
$$

$0 \leq \alpha^{-}=$const $<1$, uniformly in $a \leq a_{0}$,

$$
\int_{a}^{a+y}\left(u_{0}(x)-u^{+}\right) d x=O\left(y^{\alpha^{+}}\right) \text {as } y \rightarrow+\infty,
$$

$0 \leq \alpha^{+}=$const $<1$, uniformly in $a \geq a_{0}$. Let $\alpha=\max \left\{\alpha^{-}, \alpha^{+}\right\}$. Then there exists a constant $T>0$ such that

$$
|u(t, x)-\tilde{u}(t, x)| \leq C \cdot t^{-\left(1-\frac{1}{2-\alpha}\right)}, C=\text { const }>0
$$

for $t>T$ and $x \in R^{1}$, where $\tilde{u}(t, x)=S(t, x)$, if $u^{-}>u^{+}$and $\tilde{u}(t, x)=H(x / t)$, if $u^{-}<u^{+}$.

Remark. P.-D.Lax [6] in the case $u^{-}=u^{+}=\bar{u}$ proved that $u(t, x) \rightarrow \bar{u}$ as $\quad t \rightarrow+\infty t \rightarrow+\infty \quad$ uniformly in $x \in R^{1}$. C.M. Dafermos [7] proved, that if

$$
\int_{a}^{a+y}\left(u_{0}(x)-\bar{u}\right) d x=O\left(y^{\alpha}\right)
$$

as $y \rightarrow \infty, 0 \leq \alpha=$ const $<1$, uniformly in $a \in R^{1}$, then $|u(t, x)-\bar{u}|=O\left(t^{-\left(1-\frac{1}{2-\alpha}\right)}\right)$ as $t \rightarrow+\infty$ uniformly in $x \in R^{1}$.
The proofs of the theorems 1-3 are based on the explicit representation of the generalized solutions of the problem (1), (2) (see [5], [6]). Assume at first that $f^{\prime \prime}(u)>\varepsilon=$ const $>0$ for $u \in R^{1}$. For $(t, x) \in \Pi^{+}$, $y \in R^{1}$ consider the functional

$$
I(t, x, y)=\int_{0}^{y} u_{0}(x) d x+t \Phi\left(\frac{x-y}{t}\right)
$$

Here the function

$$
\Phi(q)=\max _{p \in \llbracket}\{p q-f(p)\}=q f^{\prime-1}(q)-f\left(f^{\prime-1}(q)\right)
$$

is a Legendre transform of the function $f(p)$. Since $f(p) /|p| \rightarrow+\infty$ as $|p| \rightarrow+\infty$, then the function $\Phi(q)$ is finite $\forall q \in R^{1}, \Phi(q) \in C^{2}\left(R^{1}\right), \Phi^{\prime \prime}(q)>0$,

$$
\Phi^{\prime}(q)=f^{\prime-1}(q), \Phi(q) / q \rightarrow+\infty \text { as } q \rightarrow+\infty .
$$

Introduce the set

$$
Y(t, x)=\left\{y \in R^{1}: I(t, x, y)=\inf _{s \in R^{1}} I(t, x, s)\right\} .
$$

This set is compact, because the functional $I(t, x, y)$ is continuous for all its arguments and $I(t, x, y) \rightarrow+\infty$ as $|y| \rightarrow+\infty$ for fixed point $(t, x)$. Denote by

$$
y_{*}(t, x)=\min Y(t, x), y^{*}(t, x)=\max Y(t, x) .
$$

Lemma([5], [6]). If $x_{1}<x_{2}$ then

$$
y^{*}\left(t, x_{1}\right) \leq y_{*}\left(t, x_{2}\right) \forall t>0 .
$$

Corollary. The functions $y_{*}(t, x)$ and $y^{*}(t, x)$ are monotonically non-decreasing in $x$ for each fixed $t>0$, $y_{*}(t, x)$ is left semi-continuous in $x, y^{*}(t, x)$ is right semi-continuous in $x$ and booth functions have one and the same no more than countable set of points of discontinuity and coincide everywhere outside this set.

The generalized solutions of the problem (1), (2) is given by a formula ([5], [6])

$$
u(t, x)=f^{\prime-1}\left(\frac{x-y^{*}(t, x)}{t}\right) .
$$

Note, that $|u(t, x)| \leq M$ if $\left|u_{0}(x)\right| \leq M$. Therefore it is enough to know the behavior of the function $f(u)$ in $[-M, M]$ only. Consequently we shall always assume $f^{\prime \prime}(u)>\varepsilon=$ const $>0 \forall u \in R^{1}$, where $\varepsilon=\min _{[-M, M]} f^{\prime \prime}(u)$, having changed $f(u)$ outside $[-M, M]$ whenever necessary.

Proof of theorem 3. We use the notations 


$$
\begin{gathered}
U_{0}^{ \pm}(y)=\int_{0}^{y}\left(u_{0}(x)-u^{ \pm}\right) d x, \\
y^{ \pm}(t, x)=x-f^{\prime}\left(u^{ \pm}\right) t, \\
\eta^{ \pm}(t, x)=\left[y^{ \pm}(t, x)-y^{*}(t, x)\right] / t
\end{gathered}
$$

We rewrite the representation (11) as

$$
u(t, x)=f^{\prime-1}\left(\eta^{ \pm}(t, x)+f^{\prime}\left(u^{ \pm}\right)\right) .
$$

Let $y^{ \pm}=y^{ \pm}(t, x)$ for brevity. Consider the difference

$$
\begin{aligned}
& I\left(t, x, y^{ \pm}\right)-I(t, x, y)=U_{0}^{ \pm}\left(y^{ \pm}\right)-U_{0}^{ \pm}(y)- \\
& -t\left[\Phi\left(f^{\prime}\left(u^{ \pm}\right)+\theta^{ \pm}\right)-\right. \\
& \left.-\Phi\left(f^{\prime}\left(u^{ \pm}\right)\right)-\Phi^{\prime}\left(f^{\prime}\left(u^{ \pm}\right)\right) \cdot \theta^{ \pm}\right] \equiv \\
& \equiv U_{0}^{ \pm}\left(y^{ \pm}\right)-U_{0}^{ \pm}(y)-F^{ \pm}\left(\theta^{ \pm}\right) t,
\end{aligned}
$$

Here

$$
\begin{gathered}
\theta^{ \pm}=\left(y^{ \pm}-y\right) / t \\
F^{ \pm}(\theta)=\Phi\left(f^{\prime}\left(u^{ \pm}\right)+\theta\right)-\Phi\left(f^{\prime}\left(u^{ \pm}\right)\right)-\Phi^{\prime}\left(f^{\prime}\left(u^{ \pm}\right)\right) \cdot \theta .
\end{gathered}
$$

It easy to verify, that the functions $F^{ \pm}(\theta)$ has the following properties: 1) $\left.F^{ \pm}(0)=0 ; 2\right)\left(F^{ \pm}\right)^{\prime}(0)=0$, since $\Phi^{\prime}=f^{\prime-1}$; 3) $\left(F^{ \pm}\right)^{\prime \prime}(\theta)$ are continuous and $\left(F^{ \pm}\right)^{\prime \prime}(\theta)=\Phi^{\prime \prime}\left(f^{\prime}\left(u^{ \pm}\right)+\theta\right)>0$. Hence,

$F^{ \pm}(\theta)$ is equivalent to $\frac{1}{2} \Phi^{\prime \prime}\left(f^{\prime}\left(u^{ \pm}\right)\right) \theta^{2}$ as $\theta \rightarrow 0$.

Put $y=y^{*}(t, x)$ in (14). Then

$$
I\left(t, x, y^{ \pm}\right)-I\left(t, x, y^{*}(t, x)\right) \geq 0
$$

from the definition of $y^{*}(t, x)$. We obtain the inequality

$$
F^{ \pm}\left(\eta^{ \pm}(t, x)\right) t \leq U_{0}^{ \pm}\left(y^{ \pm}(t, x)\right)-U_{0}^{ \pm}\left(y^{*}(t, x)\right) .
$$

The estimate (7) yields that

$$
\begin{gathered}
\left|\int_{y_{1}}^{y_{2}}\left(u_{0}(x)-u^{-}\right) d x\right| \leq C^{-}\left|y_{1}-y_{2}\right|^{\alpha^{-}}, \\
C^{-}=\text {const }>0,0 \leq \alpha^{-}=\text {const }<1,\left|y_{1}-y_{2}\right| \geq \text { const }>0, \\
y_{1} \leq a_{0}, y_{2} \leq a_{0} .
\end{gathered}
$$

The estimate (8) yields that

$$
\begin{gathered}
\left|\int_{y_{1}}^{y_{2}}\left(u_{0}(x)-u^{+}\right) d x\right| \leq C^{+}\left|y_{1}-y_{2}\right|^{\alpha+}, \\
C^{+}=\text {const }>0,0 \leq \alpha^{+}=\text {const }<1,\left|y_{1}-y_{2}\right| \geq \text { const }>0, \\
y_{1} \geq a_{0}, y_{2} \geq a_{0} .
\end{gathered}
$$

Let $u^{-}>u^{+}$. Then the statement of theorem 1 is true. Assume that $x<k t+a(t)$. Then there exist $T>0$ such that $y^{-}(t, x) \leq t\left[k-f^{\prime}\left(u^{-}\right)+a(t) / t\right] \leq\left(k-f^{\prime}\left(u^{-}\right)\right) t / 2$ for $t>T$ because $a(t)=o(t), t \rightarrow+\infty$. Hence, $y^{-}(t, x) \rightarrow-\infty$ as $t \rightarrow+\infty$, since $k<f^{\prime}\left(u^{-}\right)$. As is shown in [8] $\eta^{-}(t, x) \rightarrow 0$ as $t \rightarrow+\infty$ uniformly for $x<k t+a(t)$. Therefore, $\quad y^{*}(t, x)=y^{-}(t, x)+o(t) \leq$ $\leq\left(k-f^{\prime}\left(u^{-}\right)\right) t / 4$ as $t \rightarrow+\infty$ and $y^{*}(t, x) \rightarrow-\infty$ as $t \rightarrow+\infty$. Put $y_{1}=y^{-}(t, x), \quad y_{2}=y^{*}(t, x)$ in (15) and take into account the estimate (16). We get

$$
\begin{gathered}
\frac{t}{2} \Phi^{\prime \prime}\left(f^{\prime}\left(u^{-}\right)\right)\left(\eta^{-}(t, x)\right)^{2} \leq \mathrm{const} \cdot\left|y^{-}(t, x)-y^{*}(t, x)\right|^{\alpha^{-}}, \\
\frac{t}{2} \Phi^{\prime \prime}\left(f^{\prime}\left(u^{-}\right)\right)\left(\eta^{-}(t, x)\right)^{2} \leq \mathrm{const} \cdot\left|\eta^{-}(t, x)\right|^{\alpha^{-}} \cdot t^{\alpha^{-}},
\end{gathered}
$$

whence

$$
\left|\eta^{-}(t, x)\right| \leq \mathrm{const} \cdot \mathrm{t}^{\frac{\alpha^{-}-1}{2-\alpha^{-}}}
$$

Now assume that $x>k t+a(t)$. Then there exist $T>0 \quad$ such that $y^{+}(t, x) \geq t\left[k-f^{\prime}\left(u^{+}\right)+a(t) / t\right] \geq\left(k-f^{\prime}\left(u^{+}\right)\right) t / 2$ for $t>T$ because $a(t)=o(t), t \rightarrow+\infty$. Hence, $y^{+}(t, x) \rightarrow+\infty$ as $t \rightarrow+\infty$, since $k>f^{\prime}\left(u^{+}\right)$. As is shown in [8] $\eta^{+}(t, x) \rightarrow 0$ as $t \rightarrow+\infty$ uniformly for $x>k t+a(t)$. Therefore, $y^{*}(t, x)=y^{+}(t, x)+o(t) \geq$ $\geq\left(k-f^{\prime}\left(u^{+}\right)\right) t / 4$ as $t \rightarrow+\infty$ and $y^{*}(t, x) \rightarrow+\infty$ as $t \rightarrow+\infty$. Let $y_{1}=y^{+}(t, x), y_{2}=y^{*}(t, x)$ in (15) and take into account the estimate (17). We get

$$
\left|\eta^{+}(t, x)\right| \leq \text { const } \cdot \mathrm{t}^{\frac{\alpha^{+}-1}{2-\alpha^{+}}} .
$$

The estimate (9) follows from the estimates (18) and (19) and the representation (13).

Let $u^{-}<u^{+}$. Then the theorem 2 is true. If $x \leq f^{\prime}\left(u^{-}\right) t$, then according to [8] $y^{*}(t, x)<N, N=$ const $>0$, beginning with some moment of time,$\eta^{-}(t, x) \rightarrow 0$ as $t \rightarrow+\infty$ uniformly for $x$, and $y^{-}(t, x) \leq 0$. The 
estimate (18) follows from the estimate (16) and the inequality (19). If $x \geq f^{\prime}\left(u^{+}\right) t$, then according to [8] $y^{*}(t, x)>-N, N=$ const $>0, \quad$ beginning with some moment of time, $\eta^{+}(t, x) \rightarrow 0$ as $t \rightarrow+\infty$ uniformly for $x$, and $y^{+}(t, x) \geq 0$. The estimate (19) follows from the estimate (17) and the inequality (15).

Let now $f^{\prime}\left(u^{-}\right) t \leq x \leq f^{\prime}\left(u^{+}\right) t$. Then

$$
y^{*}\left(t, f^{\prime}\left(u^{-}\right) t\right) \leq y^{*}(t, x) \leq y^{*}\left(t, f^{\prime}\left(u^{+}\right) t\right)
$$

by virtue of lemma 1 . Because

$$
\begin{gathered}
y^{ \pm}\left(t, f^{\prime}\left(u^{ \pm}\right) t\right)=0, \\
\eta^{ \pm}\left(t, f^{\prime}\left(u^{ \pm}\right) t\right)=-y^{*}\left(t, f^{\prime}\left(u^{ \pm}\right) t\right) / t,
\end{gathered}
$$

we get from (18), (19) the estimate

$$
\left|\frac{y^{*}(t, x)}{t}\right| \leq \text { const } \cdot t^{-\frac{1-\alpha}{2-\alpha}}, \alpha=\max \left\{\alpha^{-}, \alpha^{+}\right\} .
$$

The estimate (9) follows from the formula (11), the estimate (20) and the definition (6) of the function $H(x / t)$. The theorem 3 is proved.

Corollary 1. Assume that

$u_{0}(x)-u^{ \pm} \in L^{q \pm}\left(R^{ \pm}\right) \cap L^{\infty}\left(R^{ \pm}\right), q^{ \pm} \geq 1$,

$R^{ \pm}=\left\{x \in R^{1}: \pm x>0\right\}$. Put $q=\max \left\{q^{-}, q^{+}\right\}$Then $u(t, x)-\tilde{u}(t, x)=O\left(t^{-1 /(q+1)}\right), t \rightarrow+\infty$, uniformly for $x \in R^{1}$.

Corollary 2. Assume that

$u_{0}(x)-u^{ \pm}=O\left(|x|^{-\beta^{ \pm}}\right), x \rightarrow \pm \infty, \beta^{ \pm}>0, \beta^{ \pm} \neq 1$.

Then $u(t, x)-\tilde{u}(t, x)=O\left(t^{-\beta /(\beta+1)}\right), t \rightarrow+\infty$, uniformly for $x \in R^{1}, \beta=\min \left\{\beta^{-}, \beta^{+}, 1\right\}$.

Indeed, $\alpha^{ \pm}=1-1 / q^{ \pm}$in the conditions of the corollary 1 to the theorem 3 . Respectively, $\alpha^{ \pm}=1-\beta^{ \pm}$, if $0<\beta^{ \pm}<1$, and $\alpha^{ \pm}=0$, if $\beta^{ \pm}>1$, in the conditions of the corollary 2 to the theorem 3 .

The estimate (9) may be sharpened in the case $u^{-}>u^{+}$.

Theorem 4. Assume that the integrals

$$
A^{-}=\int_{-\infty}^{0}\left(u_{0}(x)-u^{-}\right) d x, \quad A^{+}=\int_{0}^{+\infty}\left(u_{0}(x)-u^{+}\right) d x
$$

exists and $u^{-}>u^{+}$(in this case $\alpha=\alpha^{-}=\alpha^{+}=0$ in (7), (8)). Then

$$
u(t, x)-S(t, x)=o\left(t^{-1 / 2}\right), t \rightarrow+\infty,
$$

uniformly for $x \in R^{1}$. If

$$
\left|\int_{y}^{ \pm \infty}\left(u_{0}(x)-u^{ \pm}\right) d x\right| \leq C^{ \pm}|y|^{-\alpha^{ \pm}},
$$

$C^{ \pm}=$const $>0, \alpha^{ \pm}=$const $>0, \pm y \geq$ const $>0$, then

$$
|u(t, x)-S(t, x)| \leq C \cdot t^{-\frac{1+\alpha}{2}},
$$

$C=$ const $>0, \alpha=\min \left\{\alpha^{-}, \alpha^{+}\right\}$, beginning with some moment of time $T$, uniformly for $x \in R^{1}$.

Proof. Obviously, $\lim _{y \rightarrow \pm \infty} U_{0}^{ \pm}(y)=A^{ \pm}$. Hence, $U_{0}^{ \pm}\left(y_{1}\right)-U_{0}^{ \pm}\left(y_{2}\right) \rightarrow 0$ as $y_{1,2} \rightarrow \pm \infty$. If $x<k t+a(t)$, then $\quad y^{-}(t, x) \rightarrow-\infty, \quad y^{*}(t, x) \rightarrow-\infty$ and $U_{0}^{-}\left(y^{ \pm}(t, x)\right)-U_{0}^{-}\left(y^{*}(t, x)\right) \rightarrow 0 \quad$ as $\quad t \rightarrow+\infty$ uniformly for $x$. We get the estimate $\eta^{-}(t, x)=o\left(t^{-1 / 2}\right) \quad$ as $t \rightarrow+\infty$ uniformly for $x<k t+a(t)$ from the inequality (15). Analogously, we derive the estimate $\eta^{-}(t, x)=o\left(t^{-1 / 2}\right)$ as $t \rightarrow+\infty$ uniformly for $x>k t+a(t)$. We obtain (22) with the aid of the representation (13).

We have $\left|U_{0}^{ \pm}\left(y_{1}\right)-U_{0}^{ \pm}\left(y_{2}\right)\right| \leq C\left(\min \left\{\left|y_{1}\right|,\left|y_{2}\right|\right\}\right)^{-\alpha^{ \pm}}$ as $\pm y_{1} \geq$ const $>0, \pm y_{2} \geq$ const $>0$, from the assumption (23). The inequalities $y^{+}(t, x) \geq\left(k-f^{\prime}\left(u^{+}\right)\right) t / 2$, $y^{*}(t, x) \geq\left(k-f^{\prime}\left(u^{+}\right)\right) t / 4$ holds for $x>k t+a(t)$, beginning with some moment of time $T$. Then $\left|U_{0}^{+}\left(y^{+}(t, x)\right)-U_{0}^{+}\left(y^{*}(t, x)\right)\right| \leq$ const $\cdot \mathrm{t}^{-\alpha^{+}}$for $t>T$. Therefore, we obtain the estimate $\left|\eta^{+}(t, x)\right| \leq C \cdot t^{-\left(1+\alpha^{+}\right) / 2}$ from (15). Analogously, the estimate $\left|\eta^{-}(t, x)\right| \leq C \cdot t^{-\left(1+\alpha^{-}\right) / 2}$ holds for $x<k t+a(t)$ and large values of time. Thus we obtain (24) with the aid of the representation (13).

Remark 1. C.M. Dafermos [7] obtained earlier the estimate (22) by others methods. He also demonstrated that $\quad a(t) \rightarrow x_{0} \quad$ as $\quad t \rightarrow+\infty$, where $x_{0}=\left(A^{+}-A^{-}\right) /\left(u^{+}-u^{-}\right)$(see also [8]).

Remark 2. If the integrals (21) exist and $u^{-}<u^{+}$, then the estimate $u(t, x)-H(x / t)=O\left(t^{-1 / 2}\right)$ is exact. Let

$$
\begin{gathered}
f(u)=u^{2} / 2, u_{0}(x)=\left\{\begin{array}{c}
-1, x<0, \\
1,0 \leq x<1, \\
0, x \geq 1
\end{array} \quad u_{1}(x)=\left\{\begin{array}{c}
-1, x<0, \\
1, x \geq 0,
\end{array}\right.\right. \\
u_{2}(x)=\left\{\begin{array}{l}
1, x<1, \\
0, x \geq 1 .
\end{array}\right.
\end{gathered}
$$


We construct the function $H(x / t)$ and the solutions $u_{1}(t, x), u_{2}(t, x)$ of the Riemann problems with initial functions $u_{1}(x), u_{2}(x)$ respectively:

$$
\begin{aligned}
& H(x / t)=\left\{\begin{array}{c}
-1, x<-t, \\
x / t,-t \leq x<0, \quad u_{1}(t, x)=\left\{\begin{array}{c}
-1, x<-t, \\
x, x \geq 0,
\end{array} \quad \text { t } t \leq x<t,\right. \\
1, x \geq t,
\end{array}\right. \\
& u_{2}(t, x)=\left\{\begin{array}{l}
1, x<t / 2+1, \\
0, x \geq t / 2+1 .
\end{array}\right.
\end{aligned}
$$

For the construction of the solution $u(t, x)$ with initial function $u_{0}(x)$ consider the interaction of the solutions $u_{1}(t, x)$ and $u_{2}(t, x)$. The lines $x=t / 2+1$ and $x=t$ intersect in the point $(2,2)$. For $t>2$

$$
u(t, x)=\left\{\begin{array}{c}
-1, x<-t, \\
x / t,-t \leq x<x(t), \\
0, x \geq x(t) .
\end{array}\right.
$$

Here $x(t)=\sqrt{2 t}$ is the solution of the equation $\frac{d x}{d t}=\frac{x}{2 t}, x(2)=2$, which express the Rankine-Hugoniot relation on the line of discontinuity:

$$
\frac{d x}{d t}=\frac{f(u(t, x(t)+0))-f(u(t, x(t)-0))}{u(t, x(t)+0)-u(t, x(t)-0)} .
$$

We find $u(t, x(t)-0)=\sqrt{2 t} / t=\sqrt{2} \cdot t^{-1 / 2}$.

Theorem 5. Let $u^{-}>u^{+}$и $\left|u_{0}(x)-u^{ \pm}\right| \leq C^{ \pm} \cdot|x|^{-\alpha^{ \pm}}$ as $\pm x>N=$ const $\geq 0, \alpha^{ \pm}=$const $>0$. Then there exist a constant $T>0$ such that $|u(t, x)-S(t, x)| \leq C \cdot t^{-\alpha}$, $C=$ const $>0, \alpha=\min \left\{\alpha^{-}, \alpha^{+}\right\}$, for $t>T$ and $x \in R^{1}$.

Proof. Let $x>k t+a(t)$. Then $y^{+}(t, x) \rightarrow+\infty$, $y^{*}(t, x) \rightarrow+\infty$ as $t \rightarrow+\infty$ uniformly in $x \in R^{1}$ (see the proof of the Theorem 4). Evaluate the right-hand side in (15). Let $\alpha^{+} \neq 1$. Then

$$
\begin{gathered}
0 \leq U_{0}^{+}\left(y^{+}(t, x)\right)-U_{0}^{+}\left(y^{*}(t, x)\right) \leq \\
\leq \operatorname{const}\left(y^{+}(t, x)\right)^{1-\alpha^{+}}\left|1-\left(1-\frac{\eta^{+}(t, x)}{\left(y^{+}(t, x) / t\right)}\right)^{1-\alpha^{+}}\right| \leq \\
\leq\left(\frac{C^{+}}{1-\alpha^{+}}\right)\left|\left(y^{+}(t, x)\right)^{1-\alpha^{+}}-\left(y^{*}(t, x)\right)^{1-\alpha^{+}}\right| \leq \\
\leq \operatorname{const}\left(y^{+}(t, x)\right)^{1-\alpha^{+}} \times
\end{gathered}
$$

$$
\begin{gathered}
\times\left|\left(1-\alpha^{+}\right) \frac{\eta^{+}(t, x)}{\left(y^{+}(t, x) / t\right)}+o\left(\frac{\eta^{+}(t, x)}{\left(y^{+}(t, x) / t\right)}\right)\right| \leq \\
\leq \text { const } \cdot\left(y^{+}(t, x) / t\right)^{-\alpha^{+}} \cdot t^{1-\alpha^{+}}\left|\eta^{+}(t, x)\right| \leq \\
\leq \text { const } \cdot t^{1-\alpha^{+}}\left|\eta^{+}(t, x)\right| .
\end{gathered}
$$

If $\alpha^{+}=1$, then

$$
\begin{gathered}
0 \leq U_{0}^{+}\left(y^{+}(t, x)\right)-U_{0}^{+}\left(y^{*}(t, x)\right) \leq \\
\leq C^{+}\left|\ln \left(y^{+}(t, x)\right)-\ln \left(y^{*}(t, x)\right)\right|= \\
=C^{+}\left|\ln \left(y^{+}(t, x)\right)-\ln \left(y^{+}(t, x)\right)\left(1-\frac{\eta^{+}(t, x)}{\left(y^{+}(t, x) / t\right)}\right)\right| \leq \\
\leq \text { const } \cdot\left|\frac{\eta^{+}(t, x)}{\left(y^{+}(t, x) / t\right)}\right| \leq \text { const } \cdot\left|\eta^{+}(t, x)\right|
\end{gathered}
$$

Indeed, $\eta^{+}(t, x) \rightarrow 0$ as $t \rightarrow+\infty$ uniformly for $x$, as is proved in [4], and $y^{+}(t, x) / t \geq\left(k-f^{\prime}\left(u^{+}\right)\right) / 2$. Hence, there exist a moment of time $T>0$ such that $0 \leq\left(y^{+}(t, x) / t\right)^{-\alpha^{+}} \leq$const for $t>T$ and $x>k t+a(t)$. We obtain from (15) that $\left|\eta^{+}(t, x)\right| \leq$ const $\cdot \mathrm{t}^{-\alpha^{+}}$, for $t>T$ and $x>k t+a(t)$. Analogously the estimate $\left|\eta^{-}(t, x)\right| \leq$ const $\cdot \mathrm{t}^{-\alpha^{-}}$holds for $x<k t+a(t)$ and large values of the time. Finally the representation (13) give us the assertion of the theorem 5 .

Remark. If $u^{-}<u^{+}, u_{0}(x)$ is monotonically nondecreasing and $u_{0}(x)-u^{ \pm}=O\left(|x|^{-\beta^{ \pm}}\right), x \rightarrow \pm \infty, \beta^{ \pm}>0$, then $u(t, x)-H(x / t)=O\left(t^{-\beta /(\beta+1)}\right) \quad$ as $\quad t \rightarrow+\infty$, $\beta=\min \left\{\beta^{-}, \beta^{+}\right\}$, uniformly for $x \in R^{1}$ (see [1]).

\section{Mixed problem}

The mixed problem is set up in [8] as follows :

$$
\begin{gathered}
u_{t}+(f(u))_{x}=0, x>0, t>0, \\
u(0, x)=u_{0}(x) \text { a.e. } x>0, \\
u(t, 0)=\bar{u}_{0}(t), t>0, \\
u(t, x-0) \geq u(t, x+0), x>0, t>0 .
\end{gathered}
$$


The initial data $u_{0}(x)$ and the boundary data $\bar{u}_{0}(t)$ are measurable and bounded. The flux function $f(u)$ is assumed to be strictly convex.The condition (27) signify that

$$
u(t, 0)=\bar{u}_{0}(t) \text { and } f^{\prime}\left(\bar{u}_{0}(t)\right) \geq 0 ;
$$$$
\text { or } f^{\prime}(u(t, 0)) \leq 0 \text { and } f^{\prime}\left(\bar{u}_{0}(t)\right) \leq 0 \text {; }
$$$$
\text { or } \quad f^{\prime}(u(t, 0)) \leq 0, \quad f^{\prime}\left(\bar{u}_{0}(t)\right) \geq 0 \quad \text { and }
$$

$f(u(t, 0)) \geq f\left(\bar{u}_{0}(t)\right)$ a.e. $t>0$.

The condition (28) is the entropy condition.

The existence and uniqueness of the solution $u(t, x)$ of the problem (25)-(28) are established in [8].

Let $\bar{u}_{0}(t)=u^{-}, u^{-}=$const .

Assume that (4) holds, $H(x / t)$ is defined in (6), $k$ and $S(t, x)$ are defined in (5).

Theorem 6. Let $u^{-}<u^{+}$. Then $u(t, x)-H(x / t) \rightarrow 0$ as $t \rightarrow+\infty$ uniformly in $x>0$.

Theorem 7. Let $u^{-}>u^{+}$. If $k<0$, then $u(t, x) \rightarrow u^{+}$as $t \rightarrow+\infty$ uniformly in $x>0$. If $k>0$, then $u(t, x)-S(t, x) \rightarrow 0$, as $t \rightarrow+\infty$ uniformly in $x>0$.

Theorem 8. Assume that

$$
\int_{a}^{a+y}\left(u_{0}(x)-u^{+}\right) d x=O\left(y^{\alpha}\right) \text { as } y \rightarrow+\infty,
$$

$0 \leq \alpha=$ const $<1$, uniformly in $a \geq a_{0} \quad \forall a_{0} \geq 0$. There exist a moment of time $T>0$ such that

$$
|u(t, x)-\tilde{u}(t, x)| \leq C \cdot t^{-\left(1-\frac{1}{2-\alpha}\right)}, x>0, t>T
$$

where $C=$ const $>0, \tilde{u}(t, x)=H(x / t)$, if $u^{-}<u^{+}$, and $\tilde{u}(t, x)=S(t, x)$, if $u^{-}>u^{+}$.

Corollary 1. Assume that

$$
u_{0}(x)-u^{+} \in L^{q}((0,+\infty)) \cap L^{\infty}((0,+\infty)), q \geq 1 .
$$

Then

uniformly in $x>0$.

In the case $u^{+}=0$ and $q=1$ this assertion is stated in [9].

Corollary 2. Assume that

$$
u_{0}(x)-u^{+}=O\left(x^{-\beta}\right), x \rightarrow+\infty, \beta>0, \beta \neq 1 .
$$

Then $u(t, x)-\tilde{u}(t, x)=O\left(t^{-\gamma /(\gamma+1)}\right), \gamma=\min \{\beta, 1\}$, as $t \rightarrow+\infty$, uniformly in $x>0$.
Indeed, $\alpha=1-1 / q$ in the conditions of the corollary 1 to the theorem 8. Respectively $\alpha=1-\beta$, if $0<\beta<1$, and $\alpha=0$, if $\beta>1$ in the conditions of the corollary 2 to the theorem 8 .

The estimate (30) may be sharpened in the case $k<0$.

Theorem 9. Assume that $k<0$ and the integral $\int_{0}^{+\infty}\left(u_{0}(x)-u^{+}\right) d x \quad$ converge $(\alpha=0$ in (29)). Then $u(t, x)-u^{+}=o\left(t^{-1 / 2}\right), t \rightarrow+\infty$, uniformly in $x>0$.

$$
\text { If }\left|\int_{y}^{+\infty}\left(u_{0}(x)-u^{+}\right) d x\right| \leq C^{+}|y|^{-\alpha^{+}}, y \geq \text { const }>0,
$$

then there exist a moment of time $T>0$ such that

$$
\left|u(t, x)-u^{+}\right| \leq C \cdot t^{-\left(1+\alpha^{+}\right) / 2}, C=\text { const }>0, t>T,
$$

uniformly in $x>0$.

Theorem 10. Assume that $k<0$ and $u_{0}(x)-u^{+}=O\left(x^{-\beta}\right), x \rightarrow+\infty, \beta=$ const $>0$. Then there exist a moment of time $T>0$ such that

$$
\left|u(t, x)-u^{+}\right| \leq C \cdot t^{-\beta}, C=\text { const }>0, t>T,
$$

uniformly in $x>0$.

The theorems 8-10 may be proved by means of explicit formula derived in [8]. This formula extend the representation of Lax - Oleinik to the solution of the mixed problem. The arguments are similar to the demonstrations in the theorems 1-5.

This work was carried out using equipment provided by the Center of Collective Use of MSUT "STANKIN".

\section{References}

1. S.N. Kruzhkov, N.S. Petrosyan, Russian Math. Surveys 42(5), 1-47 (1987)

2. C.M. Dafermos, SIAM J. Math. Anal. 45(4), 20642070 (2013)

3. G.M. Khenkin, A.A. Shananin, Functional Anal. Appl. 50(1), 61-74 (2016)

4. E.U. Panov, Math. Notes, 100(1), 113-122 (2016)

5. O.A. Oleinik, Amer. Math. Soc. Transl. S.2, 26, 95172 (1963)

6. P.-D. Lax, Comm. Pure Appl. Math. 10(4), 537-566 (1957)

7. C.M. Dafermos, Characteristics in hyperbolic conservation laws. A study of the structure and the asymptotic behavior of solutions. Heriot-Watt Symposium, v.1 (London, Pitman, 1977). Research Notes in Mathematics 17 (1977), 1-58

8. N.S. Petrosyan, Differential Equations 20, 390-395 (1984) 
9. Ph. le Floch, Math. Methods in the Applied Sciences 10, 265-287 (1988)

10. Ph.le Floch, J.C. Nedelec, Mathematical Modelling and Numerical Analysis 22(3), 469-475 (1988) 